\title{
Emergenza COVID-19: riflessioni sull'esperienza di collaborazione all'iniziativa di aiuto psicologico telematico attuata dal Ministero della Salute
}

\author{
Elena Bonassi*
}

Il Ministero della Salute ha attivato, nel periodo fine aprile - fine giugno, un numero verde al quale la popolazione poteva chiamare per problemi connessi con 1'emergenza COVID-19 e chiedere un aiuto psicologico. Le chiamate arrivavano al primo livello dell'intervento, effettuato da psicologi dell'emergenza che selezionavano le richieste da indirizzare al secondo livello, effettuato da psicoanalisti e a psicoterapeuti appartenenti alle Associazioni accreditate che avevano dato la loro disponibilità.

In questo ambito, come membro sia dell'APPIA (Associazione di Psicoterapia Psicoanalitica dell'infanzia e dell'Adolescenza) che dell'APCF (Associazione di Psicoanalisi di coppia e famiglia), si è svolta la mia esperienza.

L'intervento avveniva attraverso colloqui telefonici o telematici gratuiti, da uno a quattro, con la possibilità di un quinto colloquio a distanza di un mese dalla conclusione. A questo numero verde si sono rivolte 50.000 persone, un numero enorme, soprattutto se paragonato a quelli di altre iniziative di aiuto pur qualificate e importanti. Perché? Forse perché il Ministero, facendosene garante e presentando l'iniziativa con interventi del Ministro Speranza sulle reti televisive nazionali, ha fornito al nostro lavoro una cornice istituzionale, con le caratteristiche di una funzione paterna, che è stata un elemento importante del setting.

Lo Stato ha offerto qualcosa di diverso dalla visita presso un ambulatorio del servizio pubblico. Ha offerto un servizio aperto tra le pieghe della Comunità, una possibilità per tutti di avvicinarsi in modo anonimo e non

\footnotetext{
*Neuropsichiatra Infantile, Psicoterapeuta Psicoanalitica, didatta Scuola ASARNIA, Membro APPIA (Associazione di Psicoterapia Psicoanalitica dell'Infanzia e dell'Adolescenza), Membro EFPP (Federazione Europea di Psicoterapia Psicoanalitica); Membro AIPCF (Associazione Internazionale di Psicoanalisi di Coppia e Famiglia).

E-mail: elenabonassi8@gmail.com
} 
troppo impegnativo a quel terreno psi che spesso spaventa e suscita diffidenza. Il numero verde è diventato un semaforo verde che ha dato il via alla comunicazione di una quantità di problemi, difficoltà, paure, sintomi, angosce. Una folla, pressata dall'angoscia, chiedeva di essere ascoltata.

Ma c'è modo e modo di ascoltare. Penso a un orecchio capace di intercettare l'angoscia, a un ascolto anche di ciò che non viene detto, a un ascolto rispettoso e non sospettoso, a un ascolto esplorativo che apre alla nascita di nuovi significati.

Sto parlando dell'Ascolto Psicoanalitico come elemento specifico di questo intervento. In quei mesi vivevamo qualche cosa che non conoscevamo e che non era mai successo prima, stavamo tutti dentro una situazione drammatica la cui caratteristica principale era l'incertezza.

Una situazione che diminuisce l'asimmetria e la distanza tra il paziente e il terapeuta, tra il richiedente aiuto e l'operatore. Eravamo, e ancora siamo, tutti sulla stessa barca, con la paura di essere contagiati e di morire. Questa nostra vulnerabilità credo che i richiedenti la sentissero e, se non era eccessiva, penso abbia aiutato lo stabilirsi di una buona relazione.

Ma chi erano i richiedenti aiuto?

Per quanto riguarda l'età le richieste per i bambini sono state quasi nulle. Sarebbe interessante, ma esula dal tema di oggi, riflettere sul perché. Anche gli adolescenti più giovani non si sono quasi fatti sentire mentre diverse richieste sono giunte dai tardo adolescenti, molte dai giovani adulti e in progressione crescente dagli adulti, dalle persone di mezza età e dagli anziani.

Per quanto riguarda l'estrazione sociale c'è da dire che la gratuità dell'intervento non ha determinato la prevalenza di richieste provenienti dalle persone meno abbienti.

Ciò depone per il fatto che ad attirare le richieste fossero le qualità intrinseche dell'offerta, che la collocavano in un'area per così dire transizionale, non troppo specialistica, non patologizzante, che consentiva l'anonimato. Non è un caso che quasi tutti scegliessero il telefono e non la videochiamata.

Questa proposta creata nell'emergenza, era qualche cosa di nuovo che ha richiesto adattamenti e cambiamenti.

L'emergenza, come dice il nome, non porta solo pericoli e disastri, ma fa emergere anche potenzialità sommerse, idee nuove, risorse inaspettate, sollecita la creatività, impone variazioni del setting che non deve essere rigido, ma tuttavia deve essere rigoroso. II setting interno e la solidità della nostra formazione sono le guide che ci aiutano ad attuare cambiamenti che non snaturino la qualità del nostro operare.

Essi ci permettono di cogliere gli elementi e gli oggetti psicoanalitici nelle condizioni diverse, interne ed esterne, connesse con la situazione drammatica che tutti stiamo vivendo. Il lavoro da remoto poi indubbiamente rende, oltre che diverse, anche più difficili le cose perché ci priva del corpo e di tutte le 
informazioni comunicative che solo la sua presenza reale ci può dare.

Ma se bisogna stare chiusi in casa il significato delle cose cambia: ciò che era un limite diviene una risorsa e lo schermo del computer diventa una finestra aperta sul mondo. L'evento pandemia ha fatto irruzione nella tradizione scompaginando il modo in cui eravamo abituati a vivere, a lavorare, a rapportarci, e ci ha costretto a riorganizzarci in qualche modo nuovo. Vedevamo scritto da tutte le parti 'andrà tutto bene', chi diceva 'tutto questo ci renderà migliori', chi il contrario. Non necessariamente migliori né peggiori, penso io, ma diversi. Personalmente io sento che mi ha cambiata l'aiuto che anche io sentivo di ricevere dalle persone che ascoltavo e con le quali condividevo una pena che era anche la mia. Attendevo quelle chiamate che mi facevano sentire viva, utile e solidale; quando mi dicevano 'la ringrazio, mi è tanto utile parlare con lei', anche io provavo gratitudine e una gioia diversa, che andava al di là del normale piacere di lavorare. Nei giorni terribili in cui file interminabili di furgoni portavano via le bare, quelle voci, per quanto portassero l'angoscia della morte, erano una medicina che mi aiutava a contenerla. Questa esperienza ha ampliato la mia risonanza interiore estendendo la gamma delle note che ora posso ascoltare.

Per quanto riguarda la motivazione delle chiamate dominano gli attacchi di panico, le condotte ossessivo-fobiche e gli stati depressivi.

Ma, al di là dei sintomi inerenti l'attualità, in molti casi c'è stato il riemergere ed il riacutizzarsi di problematiche precedenti o antiche.

Le persone che mi hanno contattata sono state le più varie, una folla di persone, come tanti personaggi saliti sul palcoscenico per far sentire la loro voce, per fare la loro parte nella grande tragedia collettiva.

Vi farò ascoltare quella di Roberto di 20 anni.

Sceglie Skype perché - mi dice - così almeno ci vediamo.

Nel primo colloquio vengo a sapere che, da un paese del Sud Italia si è trasferito a P., città del Nord, per frequentarne l'Università.

Nel periodo immediatamente precedente l'esplosione della pandemia ha un primo attacco di panico con palpitazioni cardiache e difficoltà respiratorie. Successivamente torna al paese per il funerale di una zia, resta lì, bloccato dal lockdown, e viene operato per un tumore al testicolo con un intervento risolutivo che non necessita di cure ulteriori. Mi dice di avere 'pensieri catastrofici' e la paura che gli venga un infarto.

Ha sognato che suo padre avrebbe avuto un infarto e che lui, sapendolo in anticipo, lo portava in ospedale. Prosegue dicendosi molto legato alla madre, con cui si confida e che aiuta nelle faccende domestiche, mica come il fratello minore che si fa i fatti suoi.

Una madre molto ansiosa, religiosissima, che lo elegge a suo confidente.

Suo padre - dice - è presente fisicamente ma lontano mentalmente e assente nelle sue funzioni di padre, da sempre svolte dalla madre: 'mi ha insegnato tutto lei, anche ad andare in bicicletta.' 
Soffre perché suo padre lo svaluta snobbando le competenze da lui acquisite negli studi universitari che sarebbero utili nella gestione del negozio di famiglia.

La ragazza non ce l'ha, ha avuto una storia sola, a 16-17 anni.

È molto studioso. A P. abita con due ragazze, che non gli piacciono perché sono disordinate, il bagno è sempre pieno dei loro capelli, e poi sono amiche e se ne stanno tra di loro. Al paese ha gli amici di sempre, se ne è fatto qualcuno a $\mathrm{P}$. ma preferisce a stare solo. $\mathrm{O}$ forse ha difficoltà a stare con gli altri? Quando era ricoverato gli dava fastidio che tutti lo andassero a trovare. Non gli piace mostrarsi nel bisogno e in situazioni di dipendenza. 'Mi piace cavarmela da solo,' dice alla fine.

A buon intenditor poche parole. Sento simpatia per questo ragazzo che ha buttato in campo, in un denso primo colloquio, tutti gli elementi della sua nevrosi con il corollario finale del 'e non ci provi proprio ad aiutarmi.' Gli ricordo che abbiamo la possibilità di fare 4 incontri e gli chiedo se vuole continuare. Dice di sì.

Nell'incontro successivo, a distanza di una settimana, mi dice di sentirsi meglio e di avere avuto meno pensieri catastrofici. È uscito a correre con un amico, ha studiato un po' meno. Però a volte ha dei flash di cose brutte o ricordi antichi, che sono come fulmini che squarciano il cielo: un incidente d'auto, la paura della visita di controllo, la paura di non poter generare, o anche che non sarà un buon padre. Guarda le belle ragazze su Instagram e vorrebbe averne una al suo fianco ma non la cerca perché ha paura dell'insuccesso. 'Perché i miei standard sono altissimi, diventare presidente del Consiglio', dice in tono semischerzoso, 'Avere la ragazza più bella del mondo,' proseguo io, e lui 'si, proprio così, o quella o niente' Dico: 'Così si cade dall'alto... fermiamoci ai primi danni'. Fa un gran sorriso e dice che gli piace come parlo... gli piace 'fermiamoci ai primi danni'.

Nei due incontri successivi è depresso e insoddisfatto. Non riesce a studiare, non ha voglia di uscire, non ha voglia di fare niente. Pensa all'incertezza del futuro, pensa che tra dieci anni potrebbe essere morto. Se anche potesse non avrebbe voglia di tornare a P. e non sa con chi prendere l'alloggio per il prossimo anno. Io temo che non lo farà.

$\mathrm{Si}$ è iscritto all'associazione di quelli che hanno il tumore al testicolo. In autunno avrà il controllo e vive nell'incertezza su cosa gli diranno. Intanto non può andare in bicicletta. Penso al papà che non glie lo ha insegnato. Dice che non è più quello di prima, non sa chi sarà e cosa vorrà fare. Per adesso è così, nell'incertezza, ed è brutto. Mi chiede che cosa sarà dopo i nostri colloqui. Che sarà? Penso alla canzone del film L'uomo che sapeva troppo 'che serà serà, nessuno saper potrà' canta la madre e seguendo la sua voce il bambino prigioniero trova la via di uscita.

L'emergenza COVID-19 in questo caso è soltanto la punta dell'iceberg. Sotto ci sono, e subito emergono, le angosce connesse al tumore al testicolo 
che a loro volta poggiano sulla conflittualità edipica comparsa già nel primo incontro. La costruzione è crollata e con lei tutti i progetti. Roberto dice che non sa più chi sarà né cosa vorrà fare. Quale nuovo assetto originerà dallo scompaginamento provocato dall'evento traumatico? È nell'incertezza ma sa che qualcosa ci sarà, infatti non dice non so più chi sono ma dice non so più chi sarò, che cosa vorrò fare, cioè quale persona nuova diventerà.

Ma ora sa, e non è poco, che il progetto di fare tutto da solo è irrealistico.

La buona esperienza che ha potuto fare col numero verde lo ha aiutato a pensare che non è poi così male farsi aiutare ed accoglie con gratitudine le indicazioni che gli do per proseguire il suo percorso di psicoterapia.

Ma questa seduta è tanto diversa da quelle che faccio in presenza? Nella sostanza direi di no. Direi che una vicinanza emotiva ed un contatto intenso e profondo è possibile raggiungerli anche da remoto se ci sono il desiderio del paziente di comunicare e la nostra capacità ascoltare, di capire e di rispondere in modo opportuno. La seduta tradizionale di per sé non garantisce che tutto questo avvenga.

Concludendo posso dire che le telefonate non approdate a nulla e le prese in carico interrotte sono state davvero poche. Ciò non vuole dire che non ci fossero persone disperate, che non riuscivano a risollevarsi e che forse sono naufragate. E neppure che non ci saranno postumi anche gravi del trauma pandemia. Saranno forse proprio gli adolescenti a pagare il prezzo più alto. Vuole semplicemente dire che: i) la maggioranza delle persone che, avendo chiamato il numero verde sono state indirizzate al secondo livello di intervento, ne hanno tratto grande vantaggio; ii) che l'efficacia dell'intervento è stata maggiore di quella che un intervento analogo solitamente ha in tempi normali; iii) che questo vale per tutte le fasce d'età, per i giovani come per i meno giovani come per gli anziani. Indipendentemente dall'età, per molte persone la pandemia, al di là della tragedia, è diventata una opportunità per ripensare la propria vita ed i quattro colloqui, che io proponevo a cadenza settimanale, sono diventate le tappe di un percorso significativo. Un mese importante della loro vita. Un risultato al di sopra delle aspettative. La gravità della situazione, ma anche il senso di solidarietà che nasce nelle situazioni di male comune, il pericolo, ma anche la spinta vitale a superarlo, il limite dei quattro incontri ma anche il desiderio prepotente di utilizzarli al meglio, queste, e chissà quali altre cose, sono state una particolare miscela di fattori che hanno favorito l'insight e il cambiamento. E così è stato possibile mettere a fuoco le difficoltà, fare collegamenti tra passato e presente, trovare nessi e significati illuminanti.

Insieme a tante criticità gli eventi avversi mobilitano risorse vitali e la paura di perdere la vita può diventare anche una spinta potente a ripensarla, a interrogarsi sui suoi lati oscuri e sui nodi irrisolti che ostacolano il cammino.

Accanto alla sofferenza si fa vivo il sentire che non si può più rimandare, che vale la pena provare a capire qualcosa sulle radici della sofferenza e, 
chissà, magari provare anche a riparare quel tanto o quel poco che resta da vivere

La pausa nel nostro fare incessante, creata dal lockdown, per qualcuno non è diventata un vuoto incolmabile ma uno spazio dell'anima che si riempiva di ricordi, pensieri, domande, non un tempo perduto ma un tempo ritrovato.

Conflitto di interessi: l'autore dichiara che non vi sono potenziali conflitti di interessi.

Approvazione etica e consenso a partecipare: non necessario.

Ricevuto per la pubblicazione: 10 dicembre 2020

Accettato per la pubblicazione: 12 dicembre 2020 .

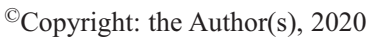

Licensee PAGEPress, Italy

Ricerca Psicoanalitica 2020; XXXI:502

doi:10.4081/rp.2020.502

This article is distributed under the terms of the Creative Commons Attribution

Noncommercial License (by-nc 4.0) which permits any noncommercial use, distribution, and reproduction in any medium, provided the original author(s) and source are credited. 